\title{
Understanding the Dynamics of Cellular Responsiveness to Modifications of Metabolic Substrates in Perifusion
}

\author{
RHONDA M. BRAND, R.H. LYONS, AND A. REES MIDGLEY* \\ Reproductive Sciences Program (R.M.B., R.H.L., A.R.M.), Bioengineering Program (R.M.B., \\ A.R.M.), Department of Biochemistry (R.H.L.), and National Center for Infertility Research \\ at Michigan (A.R.M.), the University of Michigan, Ann Arbor, Michigan 48109-0404
}

\begin{abstract}
A novel microperifusion system with capabilities for continuous, real-time, potentiometric monitoring of extracellular hydrogen ion concentration has been used to define the response of HeLa cells to abrupt changes in extracellular energy sources or introduction of an inhibitor of glycolysis. Glycolytic inhibition, induced by removal of glucose or introduction of iodoacetate, each led to a rapid, continuous decrease in acid release. The response to iodoacetate took longer than removal of glucose, perhaps due to the time required for binding and activation. Once inhibition began, however, the rate of change was greater than following glucose removal. Conversely, recovery time following iodoacetate inhibition was much slower than with glucose removal. Unlike the response to short-term glucose depletion, a second pulse of iodoacetate resulted in a faster response followed by an even longer recovery time. The response to switching between glucose and glutamine began almost without evident delay. The response patterns revealed that HeLa cells prefer glutamine to glucose, but, in the presence of both energy sources, some glucose continues to be used. In summary, these results indicate that continuous, real-time monitoring of the kinetics of hydrogen-ion release can be used to gain new insights into the dynamics of cellular response to perturbations of extracellular energy sources. (c) 1994 Wiley-Liss, Inc.
\end{abstract}

The major source of cellular energy, ATP, is generated by the oxidation of fuel molecules such as glucose, a process involving glycolysis, the tricarboxylic acid (TCA) cycle, electron transport, and oxidative phosphorylation. If cells are in an anaerobic environment or if pathways are chemically blocked, the metabolism ends after glycolysis with a different end product, lactic acid, being released. This acidifies the external environment. Alternatively, cells can use the amino acid glutamine as an energy source via glutaminolysis. Glutamine is transported into the cells where it is converted to glutamate and ammonia. Glutamate is converted to $\alpha$-ketoglutarate, a component of the citric acid cycle, from which it can enter a variety of different pathways to generate cellular energy. The ammonia released from cells upon glutamine breakdown causes the extracellular fluid to become more basic (Stryer, 1981). Lactate and ammonia are each toxic if accumulated to high levels (Glacken et al., 1986; Reuveny et al., 1986).

The release of lactate has been used often as an indicator of cellular metabolism as a few examples will indicate. Epidermal growth factor (EGF; Conricode and Ochs, 1990; Owicki et al., 1990) rapidly increases production of lactate. Stimulation of the protein kinase signalling pathways has also been demonstrated to increase the quantity of lactate released by rat hepatocytes (Conricode and Ochs, 1990) and human bone marrow cells (Wada et al., 1993). Epithelial migration
(Gibbins, 1972) has been linked to lactate release. Hyperthermia has been demonstrated to inhibit oxidative metabolism with resultant increase in lactate release by malignant but not normal cells (Overgaard, 1977). The formation of lactate and pyruvate from glucose in cultured skin fibroblasts has been proposed as a method for diagnosis of inherited diseases of the mitochondrial respiratory chain (Wijburg et al., 1990).

A number of metabolic inhibitors and uncouplers, which are able to poison electron transport and oxidative phosphorylation and thereby alter the release of lactate, have also been studied. Rotenone, cyanide, and antimycin inhibit various sites in the electron transport chain. 2,4-Dinitrophenol (DNP; Gibbins, 1972) and CCCP (Heytler and Prichard, 1962; Weinbach and Ebert, 1985) uncouple oxidative phosphorylation from the electron transport chain while malonic acid interferes with the citric acid cycle (Gibbins, 1972). The concentration of lactate is decreased by chemicals that block various glycolytic enzymes, such as iodoacetate, sodium fluoride, 2-deoxyglucose (Gibbins, 1972), mis-

Received August 6, 1993; accepted December 24, 1993.

*To whom reprint requests/correspondence should be addressed.

Dr. Rhonda M. Brand's present address is Pharmacy Department, University of California San Francisco, San Francisco, CA 941430446. 
onidazole, desmethylmisoonidazole (Chao et al., 1982), and the amino acid methionine (Boerner and Racker, 1985).

A method able to reveal the dynamics of lactate or ammonia release continuously in real time has the potential to provide a valuable tool for metabolic and pharmacologic studies. Measurements of these metabolites are often made with bioreactors, generally on a periodic, off-line, discrete sample basis (Halberstadt, 1991; Ehrlich et al., 1978). Although more difficult to interpret with complex media, because of competition between lactic acid and ammonia produced from glutamine, another approach is to focus on changes in $\mathrm{pH}$. Parce et al. have used $\mathrm{pH}$ measurements to monitor cellular metabolism on a nearly continuous basis with samples obtained at intervals on the order of minutes (Parce et al., 1989; Owicki et al., 1990; McConnell et al., 1992).

By including glucose as the only extracellular energy source available to the cells, changes in $\mathrm{pH}$ largely reflect changes in concentration of lactate. Under these conditions, approximately $80 \%$ of glucose is converted to lactate and only $8 \%$ to $\mathrm{CO}_{2}$ in HeLa cells (Rietzer et al., 1979). With such media, a perifusion system designed to reduce dispersion and miniature $\mathrm{pH}$ electrodes, we have now recorded hydrogen-ion concentrations at 1-second intervals, thereby monitoring changes in acid release effectively on a continuous basis. This system has been used to study the short-term dynamics of cellular metabolism in response to: removing glucose for brief periods (providing pulses of medium with no energy source); changing energy sources (shifting from glucose to glutamine as the only provided fuel molecule); and exposing cells to the glycolytic inhibitor iodoacetate.

\section{MATERIALS AND METHODS Perifusion system}

A Harvard apparatus compact infusion syringe pump (Model 975, Harvard, Millis MA) with a plastic $20 \mathrm{cc}$ syringe containing Hanks' balanced saline solution (HBSS) was connected to the perifusion system with a $21 \mathrm{~g}$ needle sleeved with a piece of $0.025 \mathrm{in}$. Tygon tubing with an inserted 0.012 in. Teflon tubing. Test solutions were introduced prior to the cell chamber with a six-way, low-pressure, injection valve (no. 5020, Rheodyne, Cotati, CA) with a $0.5 \mathrm{~mL}$ loop modified to reduce dead volume.

As described in detail (Brand et al., 1994), a dual electrode cell chamber was made from Plexiglas blocks of varying thickness which are stacked and clamped. The top block was drilled to hold two miniature combination electrodes (Microelectrodes, Inc., Londonderry, NH) inserted through O-rings to prevent leading. The middle 0.25 in. thick block contained a 0.1065 in. diameter hole forming a $30 \mu \mathrm{L}$ cell chamber with adjoining $12 \mu \mathrm{m}$ pore Nuclepore membranes clamped in place to constrain contained cells. The cell chamber can be accessed by a side loading port sealed by a $4-40$ stainless steel screw. The bottom block contains a small horizontal channel connecting the two vertical channels.

Test solutions contained in the injection valve loop were introduced to the side of the electrode holder as chemical signals. The medium then flowed past the first electrode, down through a small hole in the middle block, across the bottom channel, up through the bottom membrane, into the cell chamber, through the second membrane, past the second electrode, out a second side port, and then into tubing leading to waste.

A Plexiglas box with a hinged, gasketed door served as the incubator. A heating element connected to a proportional temperature controller (no. L-02156-00, Cole Parmer, Chicago, IL) operated with an YSI thermistor probe (L-08438-00, Cole Parmer) regulated the temperature (Brand et al., 1994). A blower in a styrofoam box forced air to recirculate through the heating element to the incubator and back. Temperature, monitored by a digital thermometer, remained at $37 \mathrm{C} \pm$ $0.2 \mathrm{C}$ for an 8 -hour period.

Potentials from the electrodes were amplified by electrometer units designed and constructed by KMS Fusion, Inc. (Ann Arbor, MI). Data obtained at 1-second intervals were collected via a 32-channel analog to digital converter (Opto22, Optomux, Huntington Beach, CA) connected to the Macintosh computer. All data, including the speed at which each pump is flowing at any second, were collected electronically into a spreadsheet-compatible document.

\section{Cells}

HeLa cells, obtained originally from the American Type Culture Collection, were maintained in culture by serial, trypsin-dispersed, $1: 3$ passage in $75 \mathrm{~cm}$ T-flasks using high glucose Dulbecco's modified Eagle medium $(D M E M) \pm 10 \%$ fetal calf serum (no. 320-1965-AJ, Gibco, Grand Island, NY). Prior to perifusion, $10^{6}$ cells were mixed with $200 \mu \mathrm{L}$ of a 1:1 ratio of Solohill glass beads (no. 104-1521-100, Solohill Engineering, Inc., Ann Arbor, MI) and DMEM. The mixture was transferred to one well in a 24-well microtiter plate and cultured for 1-3 days to allow cells to adhere to the beads. At experiment time, beads and cells, now in clumps, were washed and resuspended in $0.5 \mathrm{~mL}$ HBSS. One hundred microliters of the suspension was loaded into the cell chamber via the loading port, resulting in approximately $30 \mu \mathrm{L}$ of packed beads with attached cells. Upon termination of the experiment, the beads were flushed from the chamber into a $15 \mathrm{~mL}$ conical tube, incubated for 5 minutes with $1 \mathrm{~mL}$ trypsin, and counted using a hemocytometer. For the experiments described here the cell numbers per chamber ranged from $7.9 \times 10^{4}$ to $19 \times 10^{4}$.

\section{Chemicals}

HBSS with $1.0 \mathrm{~g} / \mathrm{L}$ glucose but without bicarbonate, pH 7.2-7.4 (H1387, Sigma, St. Louis, MO), was used as the basal medium. This was chosen because of its minimal buffering capacity, singular energy source, and ability to sustain cells in culture for more than 8 hours (data not shown). While the absence of bicarbonate may be thought to have a negative impact on cellular acidbase metabolism, the presence of atmospheric $\mathrm{CO}_{2}$ leads to the formation of significant levels of bicarbonate (Mason et al., 1989), and bicarbonate-free medium has been used by other investigators for many purposes as well as to study changes in extracellular acidification (Parce et al., 1989; Owicki et al., 1990; McConnell et al., 1992; Wada et al., 1993). Additionally bicarbon- 
ate-free Leibovitz L-15 medium, in which buffering is provided by amino acids and phosphate, is often used for growth of cells under conditions that permit free gaseous exchange with air.

Glucose removal was initiated by introducing pulses of HBSS without glucose, while glutamine substitution experiments replaced glucose with $4 \mathrm{mM}$ glutamine (Sigma). The two media were combined in equal parts for tests involving both substrates. The inhibitor of glycolysis, iodoacetate (Sigma), was dissolved in HBSS with glucose at a concentration of $100 \mu \mathrm{M}(7)$.

\section{Experimental procedure}

After loading, cells were allowed to equilibrate for 1 hour at a flow rate of $32 \mu \mathrm{L} / \mathrm{min}$. A 10-minute pulse of acidified HBSS (50 $\mu \mathrm{L} 0.01 \mathrm{~N} \mathrm{HCl} / 10 \mathrm{~mL}$ medium, $\mathrm{pH}$ change of $0.17 \mathrm{pH}$ unit) and basic HBSS (50 $\mu \mathrm{L} 0.01 \mathrm{~N}$ $\mathrm{NaOH} / 10 \mathrm{~mL}$ medium, $\mathrm{pH}$ change of $0.07 \mathrm{pH}$ unit) were applied as controls to insure that switching of the valve did not induce a cellular response and to obtain data necessary for analysis (see below). Ten-minute pulses of the following treatments were introduced: 1) HBSS without glucose; 2) HBSS with glutamine; 3) HBSS with glucose and glutamine; 4) HBSS with glucose applied to cells previously in a glutamine medium; and 5) HBSS plus iodoacetate. Each treatment was applied twice with a periodicity of 1 hour. Two chambers were used per experiment and each treatment was performed on two different days ( $n=8$ per treatment). The data presented are single, representative samples of results obtained from the replicates.

\section{Data analysis}

The buffering capacity of the medium was taken into account to convert changes in measured hydrogen-ion concentrations to changes in total hydrogen ions (Brand et al., 1994). In this manner, all data were converted from $\mathrm{mV}$ to femtomolar change in $\mathrm{H}^{+} /$cell-sec. All analyses were performed in the spreadsheet program Igor (Wavemetrics, Lake Oswego, OR) because of its large data set capacity and signal processing capabilities.

The high sensitivity of this measurement system (0.0005 $\mathrm{pH}$ units) effectively made it impossible to prepare test solutions with a $\mathrm{pH}$ indistinguishable from that of the control medium. Thus, in addition to the effects of cellular metabolism, the $\mathrm{pH}$ measured downstream of the cells reflects $\mathrm{pH}$ differences between the test and control media as well as the convolution of these differences with the dispersive effects of the beads, cells, chamber, and other system-related components between the pre- and post-chamber electrodes.

To predict the profile of hydrogen ions at the second electrode in the absence of other changes in hydrogenion concentration, a method of convolutional analysis was utilized (Brand, 1992). Briefly, prior to solving the convolution integral, the impulse response describing the relationship between the first and second electrodes was derived by determining the response of both electrodes to a stepwise change in $\mathrm{pH}$ and differentiating. The resulting impulse response was then subjected to fast Fourier transforms (converting to the frequency domain), the response at the second electrode divided by that of the first electrode, and inverse fast Fourier transforms performed (returning to the time domain). Once the impulse response for a particular chamber was derived, the convolution integral for the applied input signal was solved. The convolution produced the signal that would have been measured at the second (post-cellular) electrode in the absence of cellular input. This predicted response was subtracted from the measured response at that electrode. The result was termed the derived secretory profile.

The derived secretory profile was characterized in two ways: the delay until onset of response and the time to reach $50 \%$ maximum $\left(t_{50}\right)$ once the changes began. The program StatView (Brain Power Inc., Calabasas, CA) was used to perform analysis of variance as well as one-sided, unpaired t-tests which determined the reported $P$ values.

\section{RESULTS}

The need to predict the dispersive and other effects of the system on an introduced signal (e.g., a pulse of a hormone) traveling between the pre-cell chamber (first) electrode and the post-cell chamber (second) electrode provided a problem. Each run involves a unique loading of beads and cells and thus any calculated dispersive effects of the system in one perifusion run cannot be assured to be the same for another run. A test signal had to be defined that could be introduced and measured at both electrodes without perturbing the activity of the intervening cells. For this purpose, the feasibility of using a slightly acidic pulse of medium was evaluated. The results were then subjected to convolutional analysis to determine if the post-cellular measurement obtained from the acidic pulse would be predicted accurately. In Figure 1 the change in hydrogen ions measured at the pre- and post-cell electrodes, the calculated (predicted) changes in post-cell hydrogen ions, and the derived cellular responses are presented. As indicated, measured changes in hydrogen ions at the second (post-cell) electrode closely followed the changes predicted from measurements at the first electrode. The difference between these profiles, the derived secretory profile, is almost flat. The observed differences are compatible either with system variability or a very small response of the cells to slightly different $\mathrm{pH}$ pulses, a response that is much smaller than was observed following any of the experimental treatments.

To study the dynamics of response to extracellular energy deprivation, cells were denied all extracellular energy sources for 10 or 13 minutes. Since the only fuel molecule in the medium used for these experiments was glucose, this was accomplished by applying a pulse of glucose-free medium (Fig. 2, Table 1). The dynamics of the cellular response to a loss of glucose varied some between experiments. Sometimes a pattern like that shown in Figure 2A was observed, with a rapid rise followed by an overshoot above the baseline and then a rapid return to that found for basal medium. A somewhat different pattern as demonstrated in Figure 2B was also seen. Glucose removal consistently induced a small initial increase in acid release. In less than 40 seconds after the glucose-deficient pulse was received by the cells, a gradual decrease in release of hydrogen ions began (mean $=32.6$ seconds). This fall continued in a nearly linear fashion (mean $t_{50} \pm S E=173.9$ sec- 


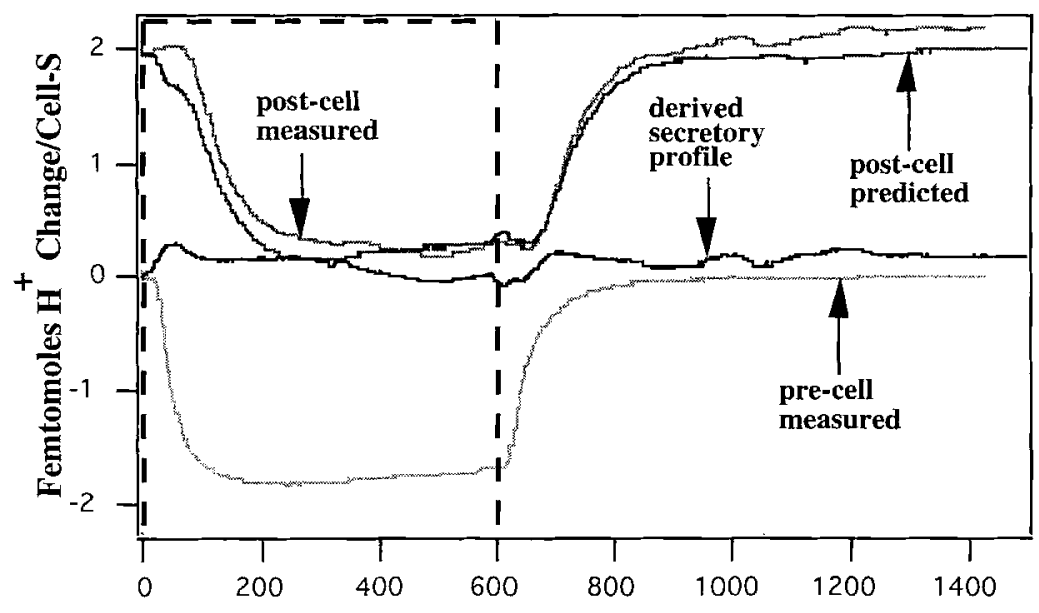

Time (s)

Fig. 1. Cellular response to HBSS with $\mathrm{NaOH}$ added. The measured responses of the pre- and post-cell electrodes, the predicted post-cell response, and the derived cellular response are presented. The derived secretory profile is equal to the post-cell measured response minus the post-cell predicted response. The rectangular box corresponds to the pulse application. The broken line brackets the pulse application. The zero value on the vertical axis indicates the relative $\mathrm{pH}$ when the experiment started, and the vertical axis indicates observed hydrogen ion changes from this initial state. onds \pm 22.9 ) until approximately 9 minutes after pulse introduction when it began to level off. Upon reintroduction of glucose, the cells began to release hydrogen ions rapidly and then gradually slowed until the original rate of acid release was reached (mean $t_{50} \pm \mathrm{SE}=$ 28.5 seconds \pm 4.7 ). Removal of glucose for short periods did not seem to alter the ability of the cells to respond again. When a second pulse was applied 1 hour later, the observed response profile was similar.

To evaluate the ability of the cells to convert from glucose-based to glutamine-based metabolism, a 10minute pulse of medium containing glutamine as the only extracellular fuel molecule was introduced. Ammonia released from the conversion of glutamine to $\alpha$-ketoglutarate was expected to cause the extracellular fluid to become more alkaline. The fall in protons (increase in ammonia release) leading to the re-establishment of a new baseline was faster than with glucose deprivation $\left(t_{50}=47.4 \pm 6.8\right.$ seconds vs. $173.8 \pm 27$ seconds, $P<0.05 ;$ Fig. 3 , Table 1 ). When the cells were returned to a glucose-containing medium, acid release decreased slightly prior to a rapid return to baseline. This return to baseline was slower than observed following reintroduction of glucose to glucose-free me$\operatorname{dium}\left(t_{50}=41.4 \pm 6.7\right.$ seconds vs. $28.5 \pm 4.69$ seconds, $P<0.05)$. As with the glucose deprivation experiments, no appreciable differences were observed between the first and second pulses.

The cells were then provided with a 10-minute pulse of medium containing both sources of energy (Fig. 4). The cellular response was similar to that seen solely following the introduction of glutamine. However the $\mathbf{t}_{50}$ for the combined fuels was less than seen with glutamine alone $\left(\mathrm{t}_{50}=28.3 \pm 1.74\right.$ vs. $47.4 \pm 6.8$, $P<0.05)$. The return to baseline was slower than expected with a ratio between $t_{50}$ initial response and $t_{50}$
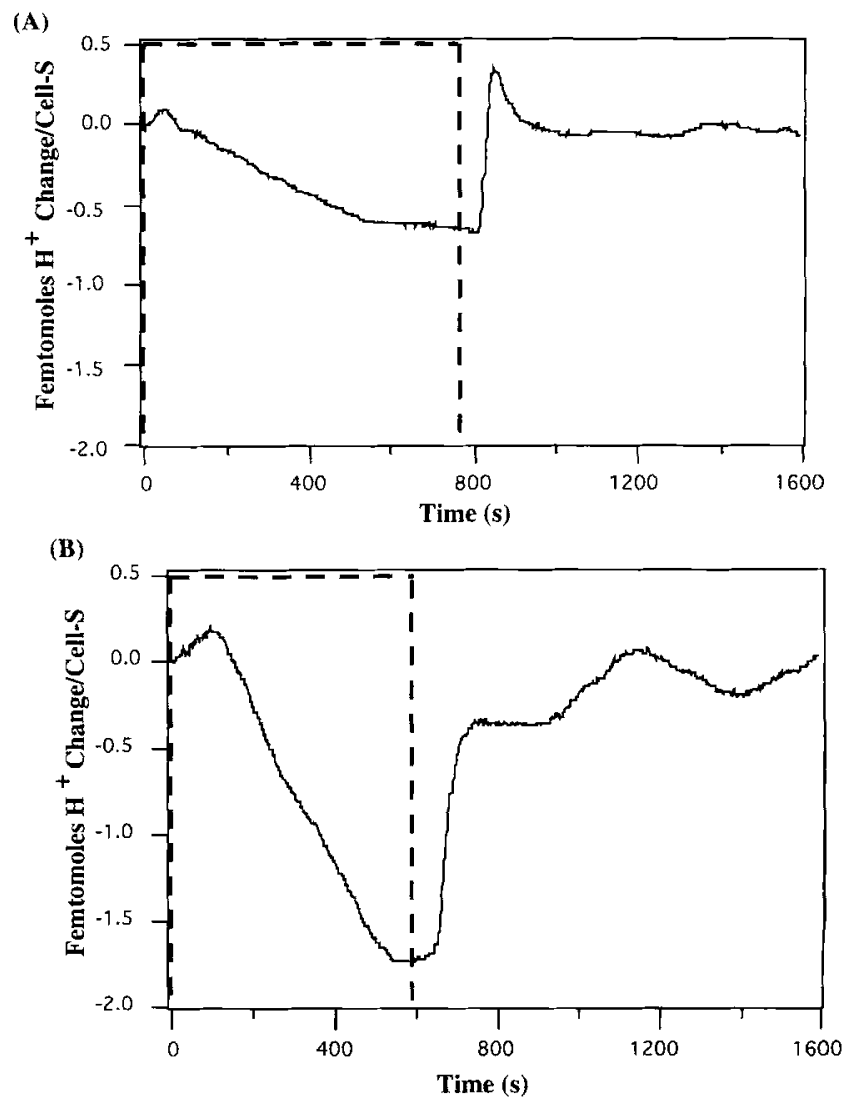

Fig. 2. Derived secretory profile to 13 minutes (A) and 10 minutes (B) of glucose deprivation. The broken line demarcates the duration of the no glucose pulse. 
TABLE 1. Summary of cellular response to various metabolic manipulations ${ }^{1}$

\begin{tabular}{lccccc}
\hline & $\begin{array}{c}\text { Glucose } \\
\text { only } \\
(\mathrm{n}=7)\end{array}$ & $\begin{array}{c}\text { Glutamine } \\
\text { only } \\
(\mathrm{n}=8)\end{array}$ & $\begin{array}{c}\text { Glucose }+ \text { glutamine } \\
(\mathrm{n}=6)\end{array}$ & $\begin{array}{c}\text { Iodoacetate } \\
(\mathrm{n}=7)\end{array}$ & $\begin{array}{c}\text { Medium } \\
(\mathrm{n}=8)\end{array}$ \\
\hline $\begin{array}{l}\text { Delay until response }(\mathrm{s}) \\
\text { (mean } \pm \mathrm{SE})\end{array}$ & $92.0 \pm 8.8$ & $63.6 \pm 2.3$ & $60.8 \pm 0.8$ & $237.6 \pm 58.0$ & $59.4 \pm 4.1$ \\
$\begin{array}{l}\text { Initial- } \mathrm{t}_{50}(\mathrm{~s})(\mathrm{mean} \pm \mathrm{SE}) \\
\text { Recovery } \mathrm{t}_{50}(\mathrm{~s})(\text { mean } \pm \mathrm{SE})\end{array}$ & $\mathbf{1 7 3 . 9 \pm 2 7 . 0}$ & $52.0 \pm 6.8$ & $28.3 \pm 1.7$ & $132.0 \pm 8.5$ & \\
\hline
\end{tabular}

${ }^{\text {I }}$ Delay is defined as the time(s) required to cause a change in hydrogen-ion concentration calculated from the derived secretory profile in all cases but the medium pulse, which was obtained directly from the post-cell electrode. The $t_{50}$ is defined as the time to reach the half maximal value once the response has begun. It was determined for the initial exposure and recovery upon removal of reach the half

(A)
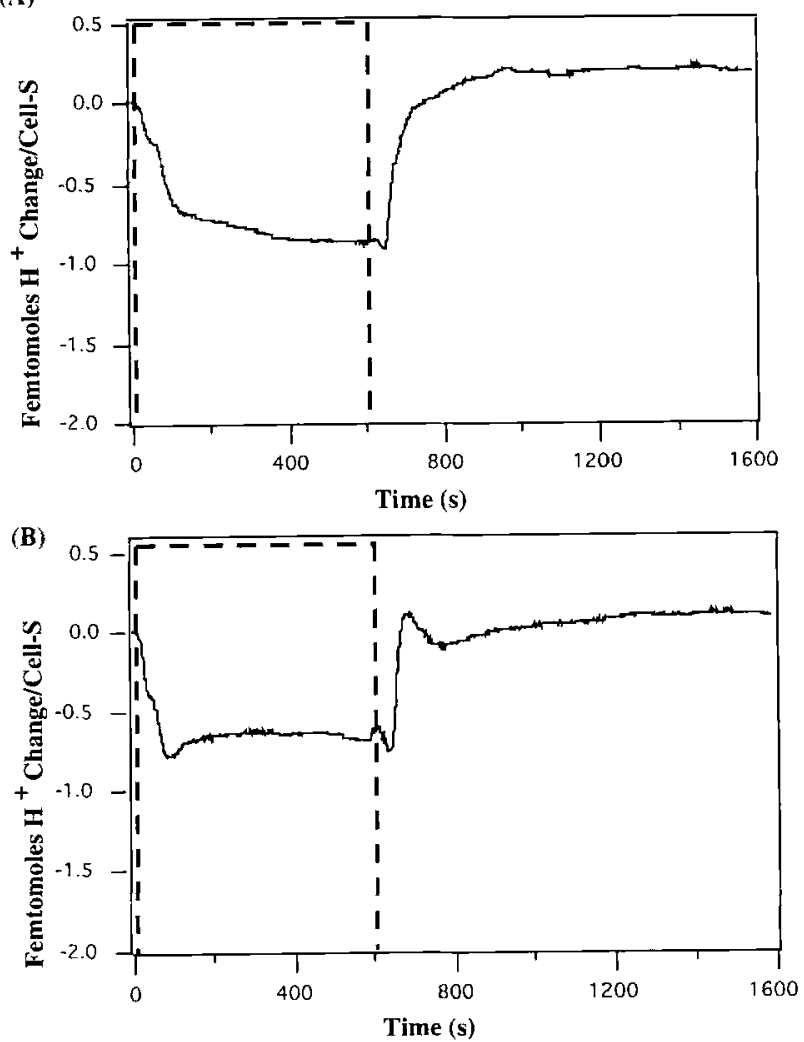

Fig. 3. Derived cellular responses to 10 minutes of $4 \mathrm{mM}$ glutamine replacing glucose as the only extracellular fuel molecule. The broken line delineates the duration of the no glucose pulse. The two graphs demonstrate variability between similar experiments.

recovery of less than 1 . In contrast, removal of glucose and providing only glutamine each gave ratios greater than 1.

When exposed to $100 \mu \mathrm{M}$ iodoacetate, the cells responded in a manner similar to that observed following the removal of glucose but with several notable differences. The cellular response took longer to begin in the presence of iodoacetate than following the removal of glucose (delay $=237.6 \pm 57.9$ seconds vs. $92.0 \pm 8.78$ seconds, $P<0.05$ ) (Table 1, Fig. 5). Once initiated, however, the response generally occurred at a greater rate $\left(t_{50}=132.0 \pm 8.5\right.$ vs. $\left.173.9 \pm 27.0, P<0.05\right)$. When iodoacetate was removed, cellular recovery was

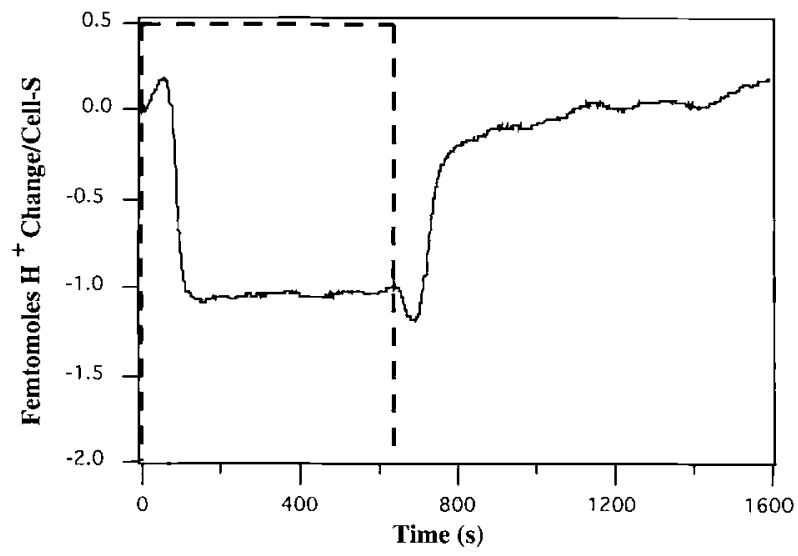

Fig. 4. Derived cellular responses to 10 minutes of combined $4 \mathrm{mM}$ glutamine and $1.0 \mathrm{~g} / \mathrm{L}$ glucose as the fuel molecules instead of only glucose. The broken line depicts the duration of the combined glucose and glutamine pulse.

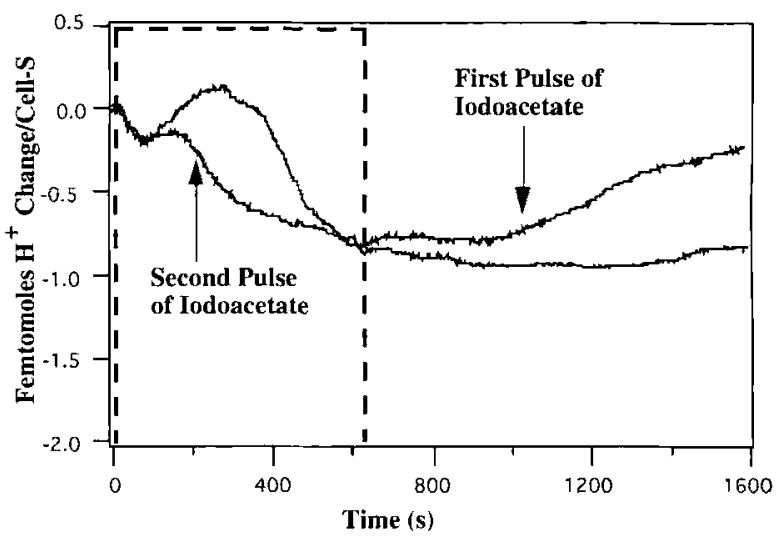

Fig. 5. Derived cellular response to 10-minute pulses of iodoacetate for two replicates separated by 1 hour. The broken line represents the duration of the iodoacetate pulse.

not immediate as with glucose deprivation but rather was slow to begin and gradual $\left(\mathrm{t}_{50}=267.3 \pm 17.8 \mathrm{vs}\right.$. $31.4 \pm 4.7, P<0.05$ ). A second pulse of iodoacetate 1 hour after the first resulted in an initial response more like that following glucose deprivation. This second response occurred faster with a decrease in acid release that was far more gradual than with the earlier pulse. 
The recovery following removal of the second pulse of iodoacetate also took longer to begin.

\section{DISCUSSION}

While the interactions of metabolic pathways over the course of days have been studied extensively (Reuveny et al., 1986; Zielke et al., 1978), little has been done to examine these relationships in the time frame of minutes. Electrodes measuring $\mathrm{pH}$, included on-line in the perifusion system, provide an excellent approach for determining one parameter of the initial dynamics of cellular responsiveness to changes in fuel molecules such as glucose and glutamine. This system can also be used to examine the influences of toxic molecules on cellular metabolism. While measurement of extracellular protons provides valuable information concerning cellular metabolism, it is only one component of biochemical dynamics. A detailed analysis of intracellular metabolism must include measurements of many other components, including $\mathrm{NADH}, \mathrm{FADH}, \mathrm{ATP}, \mathrm{NH}_{4^{+}}$, $\mathrm{CO}_{2}$, and synthesis and degradation of amino acids and lipids.

For quantitative studies of metabolism, the measurement of hydrogen-ion concentration appears to be highly suitable. Robust, hydrogen ion-selective electrodes are readily available in many sizes, are relatively inexpensive, and can be adapted to many different systems. By monitoring the concentration of released hydrogen ions, one can obtain a good general indication of overall cellular activity and response to agents such as toxins. However, the concentration of extracellular hydrogen ions can be affected by many different metabolic pathways and this can make interpretation difficult. This problem has been greatly reduced by using a cell culture medium with only a single fuel molecule, glucose. By limiting the pathways that are involved in sustaining the cells, interpretation of medium acidification becomes far simpler. Preliminary studies demonstrated that the cells could be maintained in HBSS for at least 8 hours without appreciable changes in the rate of acid release. Additionally, HBSS without bicarbonate has a buffering capacity much less than that of complete medium. When $50 \mu \mathrm{L}$ of $0.01 \mathrm{~N}$ $\mathrm{HCl}$ was added to $20 \mathrm{~mL}$ of either HBSS or Leibovitz $\mathrm{L}-15$, at similar $\mathrm{pH}$, the resulting $\mathrm{pH}$ change was approximately five times greater in HBSS than in Leibowitz L-15 (data not shown). Therefore, using HBSS with only one energy source increases the detectability of minute changes and allows for interpretation of data that would otherwise not be possible.

While most studies of cellular metabolism have been performed over the course of days or weeks, metabolic changes on a faster scale have been described. Anders et al. (1990) used fiber optic-based fluorescent probes in flowing systems to examine the immediate effects of changing metabolic states and introduction of toxins on NADH in yeast cells. Oxygen uptake was used to examine the influence of metabolic uncouplers on cellular metabolism (Gregg et al., 1968). Microcalorimetry was used to examine heat evolution from cells and to compare it with oxygen consumption (Nedergaard et al., 1977). These techniques revealed that metabolic changes induced by altering substrates, introducing toxins or uncouplers occur on time scales similar to those reported in this work. Rates of metabolic processes have also been examined in longer-term cultures of many cell lines (Thilly, 1986).

Parce et al. (1989) reported the development of a hydrogen-ion measurement system which recorded changes in external medium at approximately 6-minute intervals. Their technique is based on stopping flow for a short time, allowing hydrogen ions to accumulate and determining acidification rates. The accumulated medium is then flushed out and the cycle repeated. More recently, their sampling intervals have been reduced to between 40 seconds (McConnell et al., 1992) and 2-3 minutes (Wada et al., 1993). In contrast, the data presented here have been made with 1-second sampling intervals in the presence of continuously flowing medium. Since many of the responses examined in this study occurred in less than 1 minute, rapid sampling was important for detailed kinetic analysis; however, the overall metabolic changes due to loss of carbon source were similar for the two systems (McConnell et al., 1992).

Cells generate only 2 moles of ATP when a mole of glucose is converted to lactate, but 36 moles if a mole of glucose is completely catabolized via the TCA cycle. A mole of glutamine oxidized to lactate yields 6 moles of ATP, but if a mole of glutamine is completely converted to $\mathrm{CO}_{2}$, the yield is 21 moles of ATP. Incomplete oxidation to aspartate yields 12 moles ATP/mole of glutamine (Glacken et al., 1986). When HeLa cells were cultured in a complete medium with concentrations of glucose greater than $1 \mathrm{mM}$ about $80 \%$ of the glucose carbon was metabolized by glycolysis to lactate, $8 \%$ converted to $\mathrm{CO}_{2}$, and only 4-5\% entered the citric acid cycle (Rietzer et al., 1979). Thus, if these static culturebased data are relevant to the perifusion system and if all of the $\mathrm{CO}_{2}$ were converted to carbonic acid, at most $10 \%$ of the glucose carbon would become $\mathrm{CO}_{2}$. Conversely, HeLa cells have been reported to metabolize glutamine as follows: less than $2 \%$ incorporation into protein, 35\% into $\mathrm{CO}_{2}, 13 \%$ into lactate, and $18-25 \%$ into macromolecules (Thilly, 1986). Therefore, substitution of glutamine for glucose as the sole extracellular energy source should result in less extracellular hydrogen-ion release for two reasons: decreased lactate synthesis $(80 \%$ to $13 \%)$ and the generation of ammonia.

Conversion from glucose- to glutamine-dominated metabolism occurred without measurable delay, whereas the response to substrate removal took considerably longer, approaching 40 seconds. Switching the cells from glucose to glutamine resulted in a much faster decrease in hydrogen-ion concentration than observed following removal of glucose. The rate at which cells returned to a purely glycolytic metabolism from each of these treatments was slightly faster after glucose removal than switching to glutamine-based metabolism. When the medium was switched from glucose to glucose plus glutamine, the perifused cells appeared to prefer glutamine. These data are consistent with the results of Rietzer et al. (1979) who estimated that glutamine contributes at least $65 \%$ of the energy molecules to cells cultured in a complete medium. 
Iodoacetate, an inhibitor of the enzyme, 3-phosphoglyceraldehyde dehydrogenase, and thereby glycolysis, produced results similar to glucose removal. Iodoacetate took longer to begin inhibiting glycolysis than removal of glucose, perhaps a result of the time required for binding and activation. Once inhibition began, however, it occurred more rapidly $\left(t_{50}=132\right.$ seconds vs. 163 seconds) than observed following glucose removal. Recovery time was much slower than after glucose removal. Contrary to the substrate removal/substitution experiments, a second pulse of iodoacetate resulted in a faster response but a slower recovery.

In many of the experiments, overshoots occurred when the input signal was changed. These could be due to cellular adaptation. Another possibility is that the overshoots result from errors in the convolution. Discrepancies due to calculation would be most severe at areas of transition.

The technique of using on-line monitoring of hydrogen-ion release in a continuously flowing perifusion system is a powerful method for examining short-term metabolic perturbations. By including a second control electrode and the technique of convolution, any influence of introducing test solutions can be removed. The method is strengthened by using a medium with a single energy source, an approach that also simplifies data interpretation. It is apparent that cells are able to convert between glucose- and glutamine-dominated metabolism within seconds. Data are consistent with earlier work in long-term cultures, which demonstrated that HeLa cells prefer glutamine as their major extracellular energy source (Thilly, 1986). Additionally, the glycolytic inhibitor iodoacetate produced somewhat different results than inhibiting glycolysis via lack of substrate. Thus, this perifusion system has been demonstrated to be extremely effective in monitoring changes in extracellular acidification. The extent to which it will be useful in revealing new types of kinetic information and providing mechanistic insights remains to be ascertained.

\section{ACKNOWLEDGMENTS}

The authors would like to thank Craig Halberstadt, Bent Boving, Hal Cantor, Paul Favreau, and Mahmoud Ghazzi for their suggestions during the course of this study and assistance in the development of the perifusion system. This work was performed as part of the NICHD's National Cooperative Program on Infertility Research and supported by grants U54 HD29184 and R01 HD18018.

\section{LITERATURE CITED}

Anders, K.D., Muller, W., Scheper, T., and Buckman, A.F. (1990) Use of flourescence sensors for the monitoring of immobilized cell culture fluorescence as optical biosensors. Ann. N.Y. Acad. Sci., 589: 492-507.

Boerner, P., and Racker, E. (1985) Methionine-sensitive glycolysis in transformed cells. Proc. Natl. Acad. Sci. U.S.A., 82:6750-6754.

Brand, R.M. (1992) Analysis of dynamic changes in HeLa cell metabolism with an on-line microperifusion system, $P h D$ thesis, University of Michigan, Ann Arbor, MI, USA

Brand, R.M., Ghazzi, M.N., Rolfes-Curl, A., Cantor, H.C., and Midg- ley, A.R. (1994) Continuous, on-line hydrogen ion monitoring to study flow dynamics of perifusion systems and cellular metabolism. Am. J. Physiol.: Endo. Metab., (in press).

Chao, C.F., Subjeck, J.R., and Johnson, R.J. (1982) Nitroimidazole inhibition of lactate production in CHO cells. Int. J. Radiat. Oncol. Biol. Phys., 8:729-732.

Conricode, K.M., and Ochs, R.S. (1990) Epidermal growth factor and 12-O-tetradecanoylphorbol 13-acetate stimulate lactate production and the pentose phosphate pathway in freshly isolated rat hepatocytes. J. Biol. Chem., 265:20931-20937.

Ehrlich, K.C., Stewart, E., and Klein, E. (1978) Artificial capillary perfusion cell culture: Metabolic studies. In Vitro, 14:443-450.

Gibbins, J.R. (1972) Metabolic requirements for epithelial migration as defined by the use of metabolic inhibitors in organ culture. Exp. Cell Res., 71:329-337.

Glacken, M.W., Fleischaker, R.J., and Sinskey, A.J. (1986) Reduction of waste product excretion via nutrient control: Possible strategies for maximizing product and cell yields on serum in cultures of mammalian cells. Biotechnol. Bioeng., 28:1376-1389.

Gregg, C.T., Machinist, J.M., and Currie, W.D. (1968) Glycolytic and respiratory properties of intact mammalian cells: Inhibitor studies. Arch. Biochem. Biophys., 127:101-111.

Halberstadt, C.R. (1991) Design implementation and modeling of a transtubular bioreactor for the growth of mammalian cells, $\mathrm{PhD}$ thesis, University of Michigan, Ann Arbor, MI, USA.

Heytler, P.G., and Prichard, W.W. (1962) A new class of uncoupling agents carbonyl cyanide phenylhydrazones. Biochem. Biophys. Res. Commun., 7:272-275.

Mason, M.J., Smith, J.D., Garcia-Solo, J.D.J., and Grinstein, S. (1989) Internal $\mathrm{pH}$-sensitive sites couple $\mathrm{Cl}^{-}-\mathrm{HCO}_{3}$ - exchange to $\mathrm{Na}^{+}-\mathrm{H}^{+}$ antiport in lymphocytes. Am. J. Physiol, 256:C428-C433.

McConnell, H.M., Owicki, J.C., Parce, J.W., Miller, D.L., Baxter, G.T. Wada, H.G., and Pitchford, S. (1992) The cytosensor microphysiometer: Biological applications of silicon technology. Science, 257 : 1906-1912.

Nedergaard, J., Cannon, B., and Lindberg, O. (1977) Microcalorimetry of isolated mammalian cells. Nature, 267:518-520.

Overgaard, J. (1977) Effect of hyperthermia on malignant cells in vivo. A review and a hypothesis. Cancer, 39:2637-2646.

Owicki, J.C., Parce, J.W., Kercso, K.M., Sigal, G.B., Muir, V.C., Venter, J.C., Fraser, C.M., and McConnell, H.M. (1990) Continuous monitoring of receptor-mediated changes in the metabolic rates of living cells. Proc. Natl. Acad. Sci. USA, 87:4007-4011.

Parce, J.W., Owicki, J.C., Kercso, K.M., Sigal, G.B., Muir, V.C., Bousse, L.J., Ross, K.L., Sikic, B.I., and McConnell, H.M. (1989) Detection of cell-affecting agents with a silicon biosensor. Science, $246: 243-247$

Reuveny, S., Veldez, D., Macmillan, J.D., and Miller, L. (1986) Factors affecting cell growth and monoclonal antibody production in stirred reactors. J. Immunol. Methods, 86:53-59.

Rietzer, L.J., Wice, B.M., and Kendall, D. (1979) Evidence that glutamine, not sugar, is the major energy source for cultured HeLa cells. J. Biol. Chem., 254:2669-2676.

Stryer, L. (1981) Biochemistry. W.H. Freeman and Company, San Francisco, pp. 255-332.

Thilly, W.G. (1986) The rationale for and elements of mammalian cell technology, In: Mammalian Cell Technology. W.G. Thilly, ed. Butterworth Publishers, Boston, MA, pp 1-8.

Wada, H.G., Indelicato, S.R., Meyer, L., Kitamura, T., Miyajima, A., Kirk, G., Muir, V.C., and Parce, J.W. (1993) GM-CSF triggers a rapid, glucose dependent extracellular acidification by TF-1 cells: Evidence for sodium/proton antiporter and PKC mediated activation of acid production. J. Cell. Physiol., 154:129 138.

Weinbach, E.C., and Ebert, P.S. (1985) Effects of succinylacetone on growth and respiration of L1210 leukemia cells. Cancer Lett., 26: 253-259.

Wijburg, F.A., Feller, N., Ruitenbeek, W., Trijbels, J.M., Sengers, R.C., Schlote, H.R., Przyrembel, H., and Wanders, R.J. (1990) Detection of respiratory chain dysfunction by measuring lactate and pyruvate production in cultured fibroblasts. J. Inherited Metab. Dis., 13:355-358.

Zielke, H.R., Ozand, P.T., Tildon, J.T., Sevdalian, D.A., and Cornblath, M. (1978) Reciprocal regulation of glucose and glutamine utilization by culture human diploid fibroblasts. Cell. Physiol., 95: $41-48$. 